\title{
Ethnic inequalities in health in later life, 1993-2017: the persistence of health disadvantage over more than two decades
}

\author{
Sarah Stopforth ${ }^{1 \star}$ (D), Dharmi Kapadia ${ }^{2}$ (D), James Nazroo $^{2}$ (D) and Laia Bécares ${ }^{1}$ \\ ${ }^{1}$ Department of Social Work and Social Care, School of Education and Social Work, University of Sussex, \\ Falmer, Brighton, UK and ${ }^{2}$ Department of Sociology, School of Social Sciences, University of Manchester, \\ Manchester, UK \\ ${ }^{\star}$ Corresponding author. Email: s.stopforth@sussex.ac.uk
}

(Accepted 9 September 2021)

\begin{abstract}
Ethnic inequalities in health and wellbeing across the early and mid-lifecourse have been well-documented in the United Kingdom. What is less known is the prevalence and persistence of ethnic inequalities in health in later life. There is a large empirical gap focusing on older ethnic minority people in ethnicity and ageing research. In this paper, we take a novel approach to address data limitations by harmonising six nationally representative social survey datasets that span more than two decades. We investigate ethnic inequalities in health in later life, and we examine the effects of socio-economic position and racial discrimination in explaining health inequalities. The central finding is the persistence of stark and significant ethnic inequalities in limiting long-term illness and self-rated health between 1993 and 2017. These inequalities tend to be greater in older ages, and are partially explained by contemporaneous measures of socio-economic position, racism, and discrimination. Future data collection endeavours must better represent older ethnic minority populations and enable more detailed analyses of the accumulation of socio-economic disadvantage and exposure to racism over the lifecourse, and its effects on poorer health outcomes in later life.
\end{abstract}

Keywords: ethnicity; health inequalities; later life; socio-economic disadvantage; racism and discrimination

\section{Introduction}

Ethnic inequalities in health and wellbeing across the early and mid-lifecourse have been well-documented in the United Kingdom (UK) (Nazroo, 2001b). People from minoritised ethnic groups tend to have much poorer health outcomes over the lifecourse than the white majority group. Ethnic inequalities are clearly observable in the early years and over childhood, e.g. in birthweight (Kelly et al., 2009), asthma 
(Panico et al., 2007), obesity (Martinson et al., 2012), and early development (Dearden and Sibieta, 2010). Ethnic inequalities are also well-established into adulthood across a range of health outcomes, in general health measures such as self-rated health and limiting long-term illness (LLTI) (Evandrou, 2000; Harding and Balarajan, 2000; Nazroo, 2001b; Bécares, 2015; Darlington et al., 2015; Evandrou et al., 2016), specific conditions such as heart disease, hypertension and diabetes (Bhopal et al., 2002; Nazroo, 2003, 1997), mental health and wellbeing (Wallace et al., 2016), and in life expectancy and healthy life expectancy (Wohland et al., 2015).

What is less known is the prevalence and persistence of ethnic inequalities in health in older age (Evandrou et al., 2016; Watkinson et al., 2021). The few crosssectional studies which have examined this have observed significant health inequalities for older ethnic minority people compared with the white majority group (Evandrou, 2000; Evandrou et al., 2016; Watkinson et al., 2021), but there is a scarcity of evidence on the persistence of inequalities over time. This is highly problematic, because the increasing population of older ethnic minority people will be a key demographic change over the next decades in Britain (see Lievesley, 2010; Victor et al., 2012).

Explanations for ethnic inequalities in health are complex, but are largely driven by inequalities in socio-economic position. People from some minoritised ethnic groups are disproportionately disadvantaged on a number of socio-economic axes, e.g. living in more disadvantaged areas (Jivraj and Khan, 2015); having poorer housing quality, insecure tenures or greater overcrowding (Finney and Harries, 2013; Shankley and Finney, 2020); having higher rates of unemployment or underemployment (Kapadia et al., 2015; Clark and Shankley, 2020) with cumulative, negative effects over the lifecourse ( $\mathrm{Li}$ and Heath, 2020); working in less advantaged, lower paid occupations (Brynin and Longhi, 2015); and often having more advantaged education levels which are not converted into corresponding occupational advantage (Zwysen and Longhi, 2018). Studies have shown that there are direct and adverse effects of socio-economic inequalities on people's physical and mental health and wellbeing (Bartley et al., 2004; Marmot, 2010, 2020; Scambler, 2012; Maheswaran et al., 2015); ethnic inequalities in socio-economic position directly relate to ethnic inequalities in health.

Racism has been found to be a key direct and indirect driver of ethnic inequalities in health. Studies report a clear association between racial discrimination and detrimental physical health, mental health, and wellbeing outcomes (Karlsen and Nazroo, 2002; Bécares et al., 2009; Wallace et al., 2016; Hackett et al., 2020), independently of socio-economic position (Nazroo, 2003). Racism has a direct impact on health and wellbeing through several mechanisms, e.g. through stress pathways, physiological reactions such as hypertension or cardiovascular problems, or negative self-esteem and wellbeing (Karlsen and Nazroo, 2002, 2004; Hudson et al., 2013; Williams and Mohammed, 2013; Wallace et al., 2016). A key mechanism through which racism and racial discrimination indirectly and negatively impact on health is by leading to socio-economic inequalities over the lifecourse (Williams, 1999; Nazroo, 2003; Bécares et al., 2009; Gee et al., 2012, 2019; Hudson et al., 2013; Darlington et al., 2015). 


\section{Ethnic inequalities over the lifecourse: the role of accumulating disadvantage}

Ethnic inequalities in health outcomes, which are apparent in early life, increase as people age (Nazroo, 2004). One mechanism by which this occurs is through the accumulation of socio-economic disadvantage over the lifecourse (Dannefer, 2003; Kendig and Nazroo, 2016). Cumulative disadvantage experienced by minoritised ethnic people in employment, earnings, housing, and neighbourhoods are underpinned and shaped by structural racism and racial discrimination. In turn, this leads to intergenerational transmission of (dis)advantage and inequality (Bécares et al., 2015).

Accumulation of disadvantage and experience or anticipation of racial discrimination leads to 'weathering' of the health of minoritised populations (Geronimus, 1992). The weathering hypothesis relates to the earlier onset of ill-health or deterioration for many ethnic minority groups compared with the white majority group due to the accumulation of exposure to disadvantage along social and economic axes over the lifecourse (Geronimus, 1992; Forde et al., 2019). In the UK, analyses of the Fourth National Survey of Ethnic Minorities supports the weathering hypothesis, demonstrating a stark widening of ethnic inequalities in health observed for people from their mid-thirties onwards (Nazroo, 2001a). Analyses of Census data further demonstrate that people from many ethnic minority groups exhibit rates of poor health typical of the White British group who are significantly older (Stopforth et al., forthcoming).

Given the evidence of stark ethnic inequalities in health throughout childhood and into adulthood, and as socio-economic inequalities and racial discrimination persist and accumulate over the lifecourse, we would expect that ethnic inequalities in health outcomes worsen in later life. Only a handful of studies in the UK have examined ethnic inequalities specifically in later life (see Evandrou, 2000; Evandrou et al., 2016, Watkinson et al., 2021). These studies have cited the importance of racism and discrimination in explaining ethnic health inequalities, but measures of racism have not been employed in the analyses. Importantly, there is limited evidence on the extent of ethnic health inequalities, and whether they persist over time. This is largely due to the neglect of older ethnic minority people in both ethnicity and ageing research (Phillipson, 2015; Torres, 2015; Bécares et al., 2020). In addition, existing datasets do not collect large enough samples to conduct robust analyses examining the circumstances of older people within specific ethnic minority groups over time and across cohorts (Bécares et al., 2020).

In the present study, we take a novel approach to address data limitations by analysing cross-sectional data from a series of nationally representative social surveys spanning more than two decades. We investigate the prevalence of ethnic inequalities in health in later life, and we examine the effects of socio-economic position and experienced racial discrimination in explaining health inequalities. We address the following research questions:

(1) What is the prevalence of ethnic inequalities in health in later life?

(2) To what extent do these inequalities persist over time? 
(3) What are the respective contributions of socio-economic position and racism in explaining ethnic inequalities in health?

(4) Do ethnic inequalities in health increase, decrease or stay the same in older ages?

We hypothesise that the importance of accumulation of disadvantage over the lifecourse will lead to sizeable ethnic inequalities in health in older ages. We theorise that accounting for socio-economic position, racism, and discrimination will attenuate ethnic inequalities as these are key drivers of ethnic inequalities in health. However, in the absence of suitable longitudinal data which adequately capture lifecourse exposure and accumulation of socio-economic inequality and experienced racial discrimination, we can only indirectly test this hypothesis using contemporaneous measures collected within cross-sectional surveys. We return to this issue in the conclusion.

\section{Data and measures}

We analyse six datasets with data collections spanning more than 20 years (19932017): the Fourth National Survey of Ethnic Minorities 1993/94 (Berthoud et al., 1997), the Health Survey for England 1999 (National Centre for Social Research and UCL Department of Epidemiology and Public Health, 2010a), the Health Survey for England 2004 (National Centre for Social Research and UCL Department of Epidemiology and Public Health, 2010b), the Citizenship Survey 2007 (Department for Communities and Local Government et al., 2019), and Understanding Society Wave 1 2009/11 and Wave 7 2015/17 (University of Essex, Institute for Social and Economic Research, 2020). ${ }^{1}$ All of the datasets have complex, multi-stage, stratified random sample survey designs and are nationally representative of either England (Health Survey for England), England and Wales (Fourth National Survey of Ethnic Minorities and Citizenship Survey) or the UK (Understanding Society). Each survey deliberately over-samples ethnic minority respondents (more information on the design of each survey is provided in the online supplementary material). Each survey further contains adequate, comparable measures to analyse ethnic health inequalities and their hypothesised determinants. The analytical samples in this paper consist of respondents aged 40 and over living in England. We focus on people aged 40 and over to reflect the earlier onset of disease and ill-health for many people from ethnic minority groups (see Nazroo, 2001a).

We examine two health outcomes: LLTI and self-rated health. For LLTI, respondents were asked whether they had any long-standing illness and if this limited their abilities to undertake typical, moderate or day-to-day activities. ${ }^{2}$ We dichotomise any LLTI compared with none (reference category is no LLTI). In each survey, respondents were also asked to assess their general health on a five-point Likert scale. The exact wording of the questions and response options differ slightly between surveys, but we dichotomise excellent, very good or good health compared with fair, poor/bad or very poor/very bad health (reference category is excellent, very good or good health). Further details on the questions and answer options for both measures in each dataset are provided in Table S1 in the online supplementary material. 
Ethnicity is self-reported in all surveys from a pre-defined list of ethnic groups. Our analyses are based on eight main groups comprising White/White British, Irish, Black Caribbean, Black African, Indian, Pakistani, Bangladeshi, and Chinese respondents (reference category is White/White British). The ethnicity variables differ slightly between surveys. In the Fourth National Survey of Ethnic Minorities and 1999 Health Survey for England, Black African people were not sampled. In the 1999 and 2004 Health Survey for England, White British is not distinct from White minority groups, although Irish respondents were identified. In our regression models, we adjust for age, age-squared (to capture non-linear effects), and sex. Age and age-squared are centred and included as continuous variables, and sex is a categorical variable comprising men and women (reference category is men).

We harmonise measures of household income, individual education level, and household National Statistics Socio-Economic Classification (NS-SEC) as closely as possible in each dataset, to reflect socio-economic position. We measure income in quintiles, based on gross household equivalised income using the Organisation for Economic Co-operation and Development modified scale (see chapter 3 in Office for National Statistics, 2015) (reference category is the highest quintile). For Understanding Society, income is provided as a continuous measure, but in all other surveys income is collected through categorical measures of income brackets. We use the mid-point of these income brackets to derive our measure. A measure of household income is asked in all surveys except the Citizenship Survey, where we create a pseudo household measure combining income information from both the respondent and partner. In each survey, we use the derived quintile measure (i.e. relative position within the survey context) rather than an absolute measure due to the difference in measurement in the original data collection exercises in each survey.

Education level is measured as the highest education qualification of the respondent and dichotomised between degree-level education and less than degreelevel education (reference category is degree-level education). In the 1999 and 2004 Health Surveys for England and the Citizenship Survey, degree-level education includes equivalent vocational qualifications such as National Vocational Qualification (NVQ) Levels 4 and 5. This is not the case in Understanding Society, where NVQ Levels 3-5 are aggregated together and so cannot be included as a degree-level equivalent.

Social class is measured using the NS-SEC as a three-category measure of managerial and professional, intermediate, and routine and manual occupations at the household level (Rose and Pevalin, 2003) (reference category is managerial and professional occupations). ${ }^{3}$ For household social class, we take the social class of the household reference person where available. In the 1999 Health Survey for England there is a head of household indicator, which is similar to a household reference person but typically defaults to the oldest male in the household. In the Fourth National Survey of Ethnic Minorities, we create a pseudo household measure by taking the most advantaged social class position out of the respondent and their partner, as there is no indicator for household reference person or head of household. In the Fourth National Survey of Ethnic Minorities and 1999 Health Survey for England, NS-SEC is not deposited, but we derive this from deposited Standard Occupational Classification codes and employment status. 
Measures of racism and racial discrimination are not collected consistently in each dataset, and are only available in the Fourth National Survey of Ethnic Minorities, the Citizenship Survey, and Understanding Society. In Understanding Society, measures of experienced racial discrimination are only available for a subset of respondents (the extra five-minute sample, alternate waves only). In the Fourth National Survey of Ethnic Minorities, respondents are asked whether they have been the victim of physical attacks, property attacks, or verbal abuse in the past 12 months due to their race or skin colour. We aggregate and dichotomise these measures to capture whether the respondent has experienced any form of racist attack or abuse (reference category is none). Respondents are also asked whether they fear racial harassment and the extent: no, not very much, a fair amount, or a great deal (reference category is no). Finally, respondents who stated they do fear racial harassment are asked whether they have avoided a series of places or scenarios due to racial harassment in the past two years, which we dichotomise as yes or no (reference category is no). In the Citizenship Survey, respondents are asked whether they are worried about physical attacks due to skin colour, ethnic origin or religion: not at all worried, not very worried, fairly worried, very worried, and don't know (reference category is not at all). In Understanding Society, respondents in the extra five-minute sample are asked a series of measures about whether they have felt unsafe, avoided, been insulted, or been attacked in a list of places, and reasons for this. We combine ever been insulted or attacked due to ethnicity, nationality or religion as a measure of experienced racist abuse, and we combine ever felt unsafe or avoided places due to ethnicity, nationality or religion as a measure of anticipation of racial harassment. We dichotomise each measure as yes or no (reference category is no).

We harmonise measures as closely as possible across the six datasets. We further include categories of missingness for the socio-economic position and racism measures to retain sample size and statistical power (see Table S2 in the online supplementary material). Each socio-economic and racism measure is therefore the same for all ethnic groups within each survey. Our aim is not to compare coefficients directly across surveys where the measures have been closely, but not exactly, harmonised across surveys (i.e. the socio-economic and racism measures). Instead, our analytical focus is on the relative rates of health outcomes within each survey, in order to reflect better the nature of inequalities over time (i.e. comparisons with the white majority group). This is a pragmatic and reasonable approach to working with multiple data sources and data collection exercises.

\section{Statistical analysis}

We address our first and second research questions concurrently, by first presenting descriptive analyses of ethnic inequalities in health over time, and then estimating a series of cross-sectional logistic regression models of our health outcomes in each dataset. The initial models adjust for ethnicity, age, age-squared, and sex. To address our third research question, we additionally adjust for socio-economic position and, where measures are available, experienced racial discrimination. To assess changes in effect sizes after adjusting for socio-economic position and experienced racial discrimination, we compare the average marginal effects of LLTI and fair or 
poor self-rated health across all three models (for a discussion of comparing nested logistic regression models and methods to address this, see Karlson et al., 2012; Connelly et al., 2016; Mize et al., 2019). To address our fourth research question, we test for interaction effects of ethnicity, age, and age-squared to examine whether ethnic inequalities in health are greater in later life. We first examine this in each cross-sectional dataset separately. We then undertake an exploratory approach to overcome the restrictive sample sizes and associated statistical power, by pooling data. This enables us to explore indicative trends and underlying patterns of ethnic inequalities in later life which we might observe if we had access to adequate sample sizes of older ethnic minority people in existing datasets. The data are analysed using Stata 16 (StataCorp, 2019), adjusted for complex survey designs, and are weighted using the appropriate deposited weights in each dataset.

\section{Results}

Table 1 presents summary statistics for respondents aged 40 and over by ethnicity and survey year. ${ }^{4}$ Generally, rates of LLTI and fair or poor self-rated health are lowest for White/White British, Irish, Black African, and Chinese respondents. Rates of LLTI and fair or poor self-rated health tend to be higher for Black Caribbean and Indian respondents, and are particularly high for Pakistani and Bangladeshi respondents. White/White British respondents have the oldest mean age compared with all other ethnic groups. Black African and Chinese respondents tend to have the youngest age profiles, around 7-10 years younger than the White/White British group.

White/White British and Irish respondents have similar and generally high rates in the most advantaged socio-economic positions. Chinese respondents are consistently highly over-represented in the most advantaged socio-economic positions. By comparison, Pakistani and Bangladeshi respondents are consistently highly underrepresented in the highest income quintile and managerial and professional occupations across all survey years. Despite having much higher rates of degree-level education, Black African and Indian respondents are consistently underrepresented in the highest income quintile in all survey years. In the earlier survey years, Black Caribbean respondents tend to be more disadvantaged on all socioeconomic axes compared with the total sample.

There are no clear patterns for the prevalence of reporting experiences of racial discrimination across ethnic minority groups. Experiences of physical or verbal abuse are less commonly reported than fearing racial harassment and altering behaviour to avoid harassment, e.g. avoiding places.

Table 2 presents the results of the cross-sectional logistic regression models for LLTI by ethnicity, age, age-squared, and sex. There are clearly persisting ethnic health inequalities over time; this is particularly the case for Pakistani and Bangladeshi respondents. Pakistani respondents have statistically significant higher odds of LLTI than the White/White British group after accounting for age, age-squared, and sex in all years (ranging from odds ratio $(\mathrm{OR})=1.66,95 \%$ confidence intervals $(\mathrm{CI})=1.19-2.32$ in 2007 to $\mathrm{OR}=4.42,95 \% \mathrm{CI}=3.28-5.96$ in 2015 / 17). Bangladeshi respondents also have significantly higher odds of LLTI than the White/White British group in all years (ranging from $\mathrm{OR}=2.17,95 \% \mathrm{CI}=1.14$ - 
Table 1. Descriptive statistics by ethnicity and survey year, weighted data

\begin{tabular}{|c|c|c|c|c|c|c|}
\hline & $\begin{array}{c}\text { Fourth National } \\
\text { Survey of Ethnic } \\
\text { Minorities 1993/94 }\end{array}$ & $\begin{array}{c}\text { Health Survey } \\
\text { for England } \\
1999\end{array}$ & $\begin{array}{l}\text { Health Survey } \\
\text { for England } \\
2004\end{array}$ & $\begin{array}{l}\text { Citizenship } \\
\text { Survey } 2007\end{array}$ & $\begin{array}{c}\text { Understanding } \\
\text { Society Wave } 1 \\
2009 / 11\end{array}$ & $\begin{array}{c}\text { Understanding } \\
\text { Society Wave } 7 \\
2015 / 17\end{array}$ \\
\hline \multicolumn{7}{|l|}{ White/White British: } \\
\hline Weighted n & 1,493 & 4,206 & 49,872 & 7,110 & 21,354 & 16,813 \\
\hline Unweighted n & 1,559 & 4,287 & 3,929 & 5,262 & 19,091 & 14,992 \\
\hline LLTI $(\%, S E)$ & $47.7(0.02)$ & $34.0(0.01)$ & $33.8(0.01)$ & $26.0(0.01)$ & $26.8(0.00)$ & $32.2(0.00)$ \\
\hline Fair/poor health $(\%, S E)$ & $33.7(0.02)$ & $31.8(0.01)$ & $31.2(0.01)$ & $29.8(0.01)$ & $27.3(0.00)$ & $25.2(0.00)$ \\
\hline Age (mean, SE) & $58.7(0.35)$ & $59.4(0.27)$ & $59.2(0.29)$ & $59.2(0.22)$ & $59.5(0.13)$ & $60.8(0.15)$ \\
\hline Women $(\%, \mathrm{SE})$ & $55.7(0.01)$ & $54.7(0.01)$ & $52.5(0.01)$ & $52.6(0.01)$ & $52.4(0.00)$ & $52.5(0.00)$ \\
\hline Highest income quintile $(\%, S E)$ & $23.1(0.01)$ & $18.7(0.01)$ & $18.8(0.01)$ & $18.0(0.01)$ & $21.0(0.00)$ & $19.1(0.00)$ \\
\hline Degree education (\%, SE) & $5.5(0.01)$ & $11.3(0.01)$ & $14.6(0.01)$ & $15.9(0.01)$ & $17.2(0.00)$ & $22.6(0.00)$ \\
\hline $\begin{array}{l}\text { Managerial/professional social } \\
\text { class }(\%, \mathrm{SE})\end{array}$ & $28.7(0.02)$ & $33.7(0.01)$ & $38.4(0.01)$ & $40.8(0.01)$ & $21.3(0.00)$ & $21.9(0.00)$ \\
\hline Experienced racist attack $(\%, S E)$ & - & - & - & - & $1.3(0.00)$ & - \\
\hline Fear racial harassment $(\%, S E)$ & - & - & - & $36.9(0.01)$ & - & - \\
\hline $\begin{array}{l}\text { Avoided places due to racial } \\
\text { harassment }(\%, S E)\end{array}$ & - & - & - & - & $2.4(0.01)$ & - \\
\hline \multicolumn{7}{|l|}{ Irish: } \\
\hline Weighted n & 56 & 716 & 2,754 & 171 & 228 & 141 \\
\hline Unweighted n & 61 & 743 & 760 & 120 & 212 & 171 \\
\hline LLTI (\%, SE) & $53.1(0.05)$ & $34.2(0.02)$ & $30.3(0.02)$ & $29.0(0.05)$ & $27.7(0.03)$ & $32.8(0.05)$ \\
\hline Fair/poor health $(\%, S E)$ & $28.7(0.05)$ & $33.5(0.02)$ & $29.9(0.02)$ & $28.9(0.05)$ & $27.3(0.03)$ & $30.8(0.05)$ \\
\hline Age (mean, SE) & $55.2(2.43)$ & $56.5(0.57)$ & $57.3(0.62)$ & $57.9(1.39)$ & $59.2(1.01)$ & $59.5(1.23)$ \\
\hline
\end{tabular}




\begin{tabular}{|c|c|c|c|c|c|c|}
\hline Women $(\%, \mathrm{SE})$ & $54.2(0.04)$ & $54.1(0.02)$ & $56.8(0.02)$ & $53.5(0.06)$ & $51.1(0.03)$ & $46.5(0.05)$ \\
\hline Highest income quintile (\%, SE) & $26.2(0.04)$ & $19.1(0.02)$ & $21.8(0.03)$ & $21.2(0.05)$ & $25.4(0.03)$ & $24.2(0.04)$ \\
\hline Degree education (\%, SE) & $5.2(0.03)$ & $10.0(0.01)$ & $15.6(0.02)$ & $20.1(0.05)$ & $23.8(0.03)$ & $29.1(0.05)$ \\
\hline $\begin{array}{l}\text { Managerial/professional social } \\
\text { class }(\%, \mathrm{SE})\end{array}$ & $38.0(0.07)$ & $32.3(0.02)$ & $37.3(0.03)$ & $39.9(0.05)$ & $24.5(0.03)$ & $23.5(0.04)$ \\
\hline Experienced racist attack (\%, SE) & - & - & - & - & $0(0)$ & - \\
\hline Fear racial harassment $(\%, \mathrm{SE})$ & - & - & - & $48.7(0.06)$ & - & - \\
\hline $\begin{array}{l}\text { Avoided places due to racial } \\
\text { harassment }(\%, S E)\end{array}$ & - & - & - & - & $0(0)$ & - \\
\hline \multicolumn{7}{|l|}{ Black Caribbean: } \\
\hline Weighted $n$ & 310 & 590 & 630 & 70 & 248 & 131 \\
\hline Unweighted $n$ & 260 & 596 & 589 & 522 & 729 & 470 \\
\hline LLTI $(\%$, SE) & $48.5(0.03)$ & $37.1(0.02)$ & $36.2(0.03)$ & $27.3(0.02)$ & $31.6(0.02)$ & $37.0(0.04)$ \\
\hline Fair/poor health (\%, SE) & $52.6(0.02)$ & $51.4(0.02)$ & $44.0(0.03)$ & $38.4(0.02)$ & $34.3(0.02)$ & $31.7(0.04)$ \\
\hline Age (mean, SE) & $55.3(0.81)$ & $57.8(0.57)$ & $56.0(0.74)$ & $54.9(0.61)$ & $55.1(0.66)$ & $56.8(0.99)$ \\
\hline Women (\%, SE) & $52.3(0.04)$ & $56.2(0.02)$ & $57.2(0.02)$ & $57.1(0.03)$ & $55.3(0.02)$ & $64.1(0.03)$ \\
\hline Highest income quintile (\%, SE) & $15.5(0.03)$ & $9.9(0.01)$ & $9.5(0.02)$ & $15.8(0.02)$ & $18.5(0.02)$ & $19.9(0.03)$ \\
\hline Degree education (\%, SE) & $3.5(0.01)$ & $4.2(0.01)$ & $12.3(0.02)$ & $15.3(0.02)$ & $17.0(0.02)$ & $24.3(0.03)$ \\
\hline $\begin{array}{l}\text { Managerial/professional social } \\
\text { class }(\%, \mathrm{SE})\end{array}$ & $15.9(0.05)$ & $16.7(0.02)$ & $28.8(0.03)$ & $35.9(0.03)$ & $24.9(0.02)$ & $25.2(0.03)$ \\
\hline Experienced racist attack (\%, SE) & $10.5(0.02)$ & - & - & - & $7.3(0.01)$ & - \\
\hline Fear racial harassment $(\%, \mathrm{SE})$ & $13.4(0.02)$ & - & - & $61.1(0.03)$ & - & - \\
\hline $\begin{array}{l}\text { Avoided places due to racial } \\
\text { harassment }(\%, S E)\end{array}$ & $5.8(0.02)$ & - & - & - & $7.4(0.02)$ & - \\
\hline
\end{tabular}


Table 1. (Continued)

\begin{tabular}{|c|c|c|c|c|c|c|}
\hline & $\begin{array}{c}\text { Fourth National } \\
\text { Survey of Ethnic } \\
\text { Minorities 1993/94 }\end{array}$ & $\begin{array}{c}\text { Health Survey } \\
\text { for England } \\
1999\end{array}$ & $\begin{array}{l}\text { Health Survey } \\
\text { for England } \\
2004\end{array}$ & $\begin{array}{l}\text { Citizenship } \\
\text { Survey } 2007\end{array}$ & $\begin{array}{c}\text { Understanding } \\
\text { Society Wave } 1 \\
2009 / 11\end{array}$ & $\begin{array}{c}\text { Understanding } \\
\text { Society Wave } 7 \\
2015 / 17\end{array}$ \\
\hline \multicolumn{7}{|l|}{ Black African: } \\
\hline Weighted n & - & - & 301 & 57 & 205 & 155 \\
\hline Unweighted n & - & - & 305 & 330 & 535 & 423 \\
\hline LLTI $(\%$, SE) & - & - & $23.4(0.03)$ & $13.1(0.02)$ & $20.6(0.02)$ & $36.4(0.04)$ \\
\hline Fair/poor health (\%, SE) & - & - & $31.3(0.03)$ & $19.1(0.02)$ & $20.7(0.02)$ & $20.2(0.03)$ \\
\hline Age (mean, SE) & - & - & $49.8(0.70)$ & $48.8(0.71)$ & $48.9(0.46)$ & $51.0(1.32)$ \\
\hline Women $(\%, \mathrm{SE})$ & - & - & $53.5(0.03)$ & $48.6(0.03)$ & $52.6(0.02)$ & $55.3(0.03)$ \\
\hline Highest income quintile $(\%, S E)$ & - & - & $12.3(0.03)$ & $12.2(0.02)$ & $15.9(0.02)$ & $12.4(0.03)$ \\
\hline Degree education $(\%, \mathrm{SE})$ & - & - & $31.2(0.04)$ & $35.2(0.03)$ & $38.3(0.03)$ & $37.8(0.04)$ \\
\hline $\begin{array}{l}\text { Managerial/professional social } \\
\text { class }(\%, \mathrm{SE})\end{array}$ & - & - & $38.5(0.04)$ & $38.2(0.04)$ & $28.1(0.03)$ & $23.8(0.04)$ \\
\hline Experienced racist attack (\%, SE) & - & - & - & - & $9.2(0.02)$ & - \\
\hline Fear racial harassment $(\%, \mathrm{SE})$ & - & - & - & $63.9(0.04)$ & - & - \\
\hline $\begin{array}{l}\text { Avoided places due to racial } \\
\text { harassment }(\%, S E)\end{array}$ & - & - & - & - & $8.9(0.02)$ & - \\
\hline \multicolumn{7}{|l|}{ Indian: } \\
\hline Weighted n & 407 & 618 & 1,020 & 124 & 441 & 289 \\
\hline Unweighted $\mathrm{n}$ & 409 & 629 & 616 & 675 & 795 & 837 \\
\hline LLTI $(\%, S E)$ & $39.7(0.03)$ & $36.1(0.03)$ & $32.2(0.02)$ & $24.3(0.02)$ & $35.2(0.02)$ & $39.4(0.03)$ \\
\hline Fair/poor health (\%, SE) & $44.6(0.03)$ & $47.2(0.03)$ & $43.4(0.03)$ & $36.3(0.02)$ & $30.2(0.02)$ & $22.9(0.02)$ \\
\hline
\end{tabular}




\begin{tabular}{|c|c|c|c|c|c|c|}
\hline Age (mean, SE) & $52.8(0.54)$ & $53.4(0.61)$ & $53.8(0.61)$ & $53.8(0.60)$ & $54.1(0.54)$ & $53.5(1.08)$ \\
\hline Women $(\%, \mathrm{SE})$ & $48.0(0.06)$ & $47.7(0.02)$ & $53.7(0.01)$ & $42.6(0.02)$ & $42.2(0.01)$ & $46.0(0.02)$ \\
\hline Highest income quintile (\%, SE) & $11.1(0.03)$ & $9.3(0.02)$ & $10.2(0.02)$ & $14.3(0.02)$ & $19.8(0.02)$ & $28.1(0.04)$ \\
\hline Degree education (\%, SE) & $19.4(0.04)$ & $20.7(0.02)$ & $23.4(0.02)$ & $23.2(0.02)$ & $26.8(0.02)$ & $31.8(0.03)$ \\
\hline $\begin{array}{l}\text { Managerial/professional social } \\
\text { class }(\%, \mathrm{SE})\end{array}$ & $13.9(0.03)$ & $27.4(0.03)$ & $31.4(0.03)$ & $35.7(0.03)$ & $26.4(0.02)$ & $32.7(0.04)$ \\
\hline Experienced racist attack (\%, SE) & $7.3(0.01)$ & - & - & - & $10.0(0.01)$ & - \\
\hline Fear racial harassment $(\%, \mathrm{SE})$ & $24.4(0.03)$ & - & - & $77.9(0.02)$ & - & - \\
\hline $\begin{array}{l}\text { Avoided places due to racial } \\
\text { harassment }(\%, S E)\end{array}$ & $16.8(0.03)$ & - & - & - & $16.1(0.02)$ & - \\
\hline \multicolumn{7}{|l|}{ Pakistani: } \\
\hline Weighted n & 150 & 395 & 332 & 50 & 190 & 118 \\
\hline Unweighted $n$ & 228 & 406 & 336 & 259 & 472 & 495 \\
\hline LLTI (\%, SE) & $55.6(0.05)$ & $43.0(0.03)$ & $45.6(0.04)$ & $29.7(0.03)$ & $43.3(0.03)$ & $56.2(0.04)$ \\
\hline Fair/poor health $(\%$, SE) & $60.2(0.02)$ & $59.4(0.03)$ & $55.3(0.03)$ & $45.6(0.04)$ & $44.3(0.03)$ & $35.4(0.04)$ \\
\hline Age (mean, SE) & $51.9(0.82)$ & $51.3(0.51)$ & $53.6(0.78)$ & $51.8(0.71)$ & $52.9(1.44)$ & $52.1(0.97)$ \\
\hline Women (\%, SE) & $45.1(0.04)$ & $45.4(0.02)$ & $51.4(0.02)$ & $37.4(0.03)$ & $42.8(0.02)$ & $46.2(0.02)$ \\
\hline Highest income quintile $(\%, S E)$ & $3.3(0.02)$ & $4.5(0.02)$ & $7.4(0.02)$ & $6.2(0.02)$ & $7.9(0.02)$ & $2.1(0.01)$ \\
\hline Degree education $(\%, \mathrm{SE})$ & $9.9(0.04)$ & $11.4(0.02)$ & $14.4(0.02)$ & $20.8(0.03)$ & $22.9(0.02)$ & $26.3(0.04)$ \\
\hline $\begin{array}{l}\text { Managerial/professional social } \\
\text { class }(\%, \mathrm{SE})\end{array}$ & $3.7(0.01)$ & $12.9(0.02)$ & $19.6(0.03)$ & $22.5(0.03)$ & $14.3(0.02)$ & $15.9(0.03)$ \\
\hline Experienced racist attack $(\%, S E)$ & $9.6(0.02)$ & - & - & - & $8.7(0.02)$ & - \\
\hline Fear racial harassment $(\%, \mathrm{SE})$ & $21.9(0.05)$ & - & - & $77.8(0.03)$ & - & - \\
\hline $\begin{array}{l}\text { Avoided places due to racial } \\
\text { harassment }(\%, S E)\end{array}$ & $17.8(0.03)$ & - & - & - & $16.4(0.03)$ & - \\
\hline
\end{tabular}


Table 1. (Continued)

\begin{tabular}{|c|c|c|c|c|c|c|}
\hline & $\begin{array}{l}\text { Fourth National } \\
\text { Survey of Ethnic } \\
\text { Minorities 1993/94 }\end{array}$ & $\begin{array}{c}\text { Health Survey } \\
\text { for England } \\
1999\end{array}$ & $\begin{array}{l}\text { Health Survey } \\
\text { for England } \\
2004\end{array}$ & $\begin{array}{l}\text { Citizenship } \\
\text { Survey } 2007\end{array}$ & $\begin{array}{c}\text { Understanding } \\
\text { Society Wave } 1 \\
2009 / 11\end{array}$ & $\begin{array}{c}\text { Understanding } \\
\text { Society Wave } 7 \\
2015 / 17\end{array}$ \\
\hline \multicolumn{7}{|l|}{ Bangladeshi: } \\
\hline Weighted $n$ & 49 & 331 & 121 & 17 & 66 & 46 \\
\hline Unweighted $\mathrm{n}$ & 94 & 353 & 279 & 74 & 295 & 169 \\
\hline LLTI (\%, SE) & $58.6(0.05)$ & $48.8(0.04)$ & $47.3(0.03)$ & $34.9(0.07)$ & $41.5(0.04)$ & $51.9(0.06)$ \\
\hline Fair/poor health $(\%, S E)$ & $66.9(0.07)$ & $70.8(0.03)$ & $65.4(0.04)$ & $40.9(0.07)$ & $44.2(0.04)$ & $29.3(0.06)$ \\
\hline Age (mean, SE) & $50.7(0.63)$ & $54.0(0.68)$ & $52.6(0.82)$ & $51.3(1.25)$ & $52.8(0.70)$ & $50.8(1.55)$ \\
\hline Women $(\%, \mathrm{SE})$ & $54.6(0.07)$ & $44.4(0.02)$ & $44.6(0.02)$ & $30.5(0.07)$ & $30.0(0.03)$ & $34.0(0.05)$ \\
\hline Highest income quintile $(\%, S E)$ & $3.7(0.02)$ & $0.8(0.01)$ & $0(0)$ & $1.3(0.01)$ & $13.1(0.04)$ & $9.0(0.03)$ \\
\hline Degree education $(\%, \mathrm{SE})$ & $8.4(0.05)$ & $4.3(0.02)$ & $5.9(0.02)$ & $9.7(0.05)$ & $17.0(0.04)$ & $23.6(0.05)$ \\
\hline $\begin{array}{l}\text { Managerial/professional social } \\
\text { class }(\%, \mathrm{SE})\end{array}$ & $6.1(0.04)$ & $3.4(0.01)$ & $12.1(0.02)$ & $16.8(0.06)$ & $15.0(0.04)$ & $15.7(0.05)$ \\
\hline Experienced racist attack $(\%, S E)$ & $6.9(0.02)$ & - & - & - & $5.2(0.02)$ & - \\
\hline Fear racial harassment $(\%, \mathrm{SE})$ & $21.3(0.06)$ & - & - & $79.0(0.05)$ & - & - \\
\hline $\begin{array}{l}\text { Avoided places due to racial } \\
\text { harassment }(\%, S E)\end{array}$ & $18.3(0.06)$ & - & - & - & $6.4(0.02)$ & - \\
\hline \multicolumn{7}{|l|}{ Chinese: } \\
\hline Weighted $n$ & 65 & 360 & 147 & 13 & 53 & 45 \\
\hline Unweighted $n$ & 42 & 366 & 336 & 61 & 86 & 86 \\
\hline LLTI $(\%, S E)$ & $39.5(0.10)$ & $18.1(0.02)$ & $14.8(0.02)$ & $4.6(0.03)$ & $18.9(0.06)$ & $16.0(0.06)$ \\
\hline Fair/poor health $(\%, \mathrm{SE})$ & $38.0(0.08)$ & $34.5(0.04)$ & $33.0(0.03)$ & $28.0(0.08)$ & $14.2(0.05)$ & $18.1(0.06)$ \\
\hline Age (mean, SE) & $52.0(2.66)$ & $52.0(0.67)$ & $52.4(0.59)$ & $51.1(1.15)$ & $51.4(0.69)$ & $50.6(2.39)$ \\
\hline
\end{tabular}




\begin{tabular}{|c|c|c|c|c|c|c|}
\hline Women $(\%, \mathrm{SE})$ & $34.5(0.09)$ & $53.6(0.02)$ & $54.4(0.02)$ & $60.7(0.09)$ & $43.0(0.05)$ & $42.1(0.07)$ \\
\hline Highest income quintile (\%, SE) & $31.0(0.14)$ & $18.5(0.04)$ & $20.7(0.03)$ & $35.1(0.08)$ & $33.3(0.07)$ & $33.4(0.08)$ \\
\hline Degree education (\%, SE) & $17.4(0.04)$ & $15.6(0.03)$ & $32.5(0.03)$ & $43.3(0.08)$ & $47.5(0.07)$ & $55.7(0.08)$ \\
\hline $\begin{array}{l}\text { Managerial/professional social } \\
\text { class }(\%, \mathrm{SE})\end{array}$ & $26.6(0.11)$ & $31.7(0.04)$ & $43.4(0.04)$ & $52.9(0.08)$ & $44.0(0.07)$ & $43.4(0.09)$ \\
\hline Experienced racist attack (\%, SE) & $5.1(0.03)$ & - & - & - & $17.6(0.05)$ & - \\
\hline Fear racial harassment (\%, SE) & $12.7(0.05)$ & - & - & $70.6(0.08)$ & - & - \\
\hline $\begin{array}{l}\text { Avoided places due to racial } \\
\text { harassment }(\%, S E)\end{array}$ & $5.2(0.02)$ & - & - & - & $18.3(0.05)$ & - \\
\hline \multicolumn{7}{|l|}{ Total sample: } \\
\hline Weighted $n$ & 2,528 & 7,214 & 55,178 & 7,611 & 22,783 & 17,738 \\
\hline Unweighted $n$ & 2,653 & 7,380 & 7,150 & 7,303 & 22,215 & 17,643 \\
\hline $\operatorname{LLTI}(\%, \mathrm{SE})$ & $47.1(0.02)$ & $34.8(0.01)$ & $33.6(0.01)$ & $26.0(0.01)$ & $27.2(0.00)$ & $32.6(0.00)$ \\
\hline Fair/poor health $(\%, \mathrm{SE})$ & $40.0(0.03)$ & $38.3(0.01)$ & $31.7(0.01)$ & $30.0(0.07)$ & $27.6(0.00)$ & $25.3(0.00)$ \\
\hline Age (mean, SE) & $56.5(0.57)$ & $57.4(0.22)$ & $58.8(0.26)$ & $58.9(0.20)$ & $59.1(0.12)$ & $60.4(0.15)$ \\
\hline Women (\%, SE) & $52.8(0.01)$ & $53.1(0.00)$ & $52.7(0.01)$ & $52.3(0.01)$ & $52.0(0.00)$ & $52.4(0.00)$ \\
\hline Highest income quintile $(\%, S E)$ & $18.9(0.02)$ & $15.6(0.01)$ & $18.6(0.01)$ & $17.8(0.01)$ & $20.8(0.00)$ & $19.1(0.00)$ \\
\hline Degree education (\%, SE) & $8.1(0.02)$ & $11.3(0.01)$ & $14.9(0.01)$ & $16.4(0.01)$ & $17.7(0.00)$ & $23.0(0.00)$ \\
\hline $\begin{array}{l}\text { Managerial/professional social } \\
\text { class }(\%, \mathrm{SE})\end{array}$ & $23.0(0.03)$ & $29.0(0.01)$ & $38.0(0.01)$ & $40.5(0.01)$ & $21.5(0.00)$ & $22.1(0.01)$ \\
\hline Experienced racist attack $(\%, S E)$ & $3.3(0.01)$ & - & - & - & $6.3(0.01)$ & - \\
\hline Fear racial harassment $(\%, \mathrm{SE})$ & $7.6(0.02)$ & - & - & $38.7(0.01)$ & - & - \\
\hline $\begin{array}{l}\text { Avoided places due to racial } \\
\text { harassment }(\%, S E)\end{array}$ & $5.0(0.02)$ & - & - & - & $8.8(0.01)$ & - \\
\hline
\end{tabular}


Table 2. Logistic regression models of limiting long-term illness across survey years

\begin{tabular}{|c|c|c|c|c|c|c|}
\hline & $\begin{array}{l}\text { Fourth National } \\
\text { Survey of Ethnic } \\
\text { Minorities 1993/94 }\end{array}$ & $\begin{array}{c}\text { Health Survey } \\
\text { for England } \\
1999\end{array}$ & $\begin{array}{l}\text { Health Survey } \\
\text { for England } \\
2004\end{array}$ & $\begin{array}{l}\text { Citizenship } \\
\text { Survey } 2007\end{array}$ & $\begin{array}{c}\text { Understanding } \\
\text { Society Wave } 1 \text { 2009/ } \\
11\end{array}$ & $\begin{array}{c}\text { Understanding } \\
\text { Society Wave } 7 \text { 2015/ } \\
17\end{array}$ \\
\hline & \multicolumn{6}{|c|}{ Odds ratios (95\% confidence intervals) } \\
\hline \multicolumn{7}{|l|}{ Ethnicity: } \\
\hline \multirow[t]{2}{*}{ White/White British } & 1.00 & 1.00 & 1.00 & 1.00 & 1.00 & 1.00 \\
\hline & $(1.00,1.00)$ & $(1.00,1.00)$ & $(1.00,1.00)$ & $(1.00,1.00)$ & $(1.00,1.00)$ & $(1.00,1.00)$ \\
\hline \multirow[t]{2}{*}{ Irish } & 1.56 & 1.13 & 0.91 & 1.23 & 1.11 & 1.13 \\
\hline & $(0.87,2.78)$ & $(0.93,1.38)$ & $(0.72,1.14)$ & $(0.81,1.87)$ & $(0.78,1.58)$ & $(0.71,1.82)$ \\
\hline \multirow[t]{2}{*}{ Black Caribbean } & 1.28 & 1.22 & 1.27 & 1.27 & $1.70^{\star \star \star}$ & $1.50^{*}$ \\
\hline & $(0.97,1.69)$ & $(0.99,1.51)$ & $(0.98,1.63)$ & $(1.00,1.61)$ & $(1.41,2.06)$ & $(1.08,2.09)$ \\
\hline \multirow[t]{2}{*}{ Black African } & & & 0.88 & $0.65^{\star}$ & $1.36^{\star}$ & $1.93^{\star \star \star}$ \\
\hline & & & $(0.63,1.22)$ & $(0.44,0.97)$ & $(1.07,1.72)$ & $(1.35,2.75)$ \\
\hline \multirow[t]{2}{*}{ Indian } & 1.06 & $1.40^{\star}$ & 1.16 & 1.15 & $2.29^{\star \star \star}$ & $2.04^{\star \star \star}$ \\
\hline & $(0.74,1.52)$ & $(1.06,1.85)$ & $(0.92,1.47)$ & $(0.90,1.46)$ & $(1.90,2.76)$ & $(1.60,2.60)$ \\
\hline \multirow[t]{2}{*}{ Pakistani } & $2.30^{\star \star}$ & $2.06^{\star \star \star}$ & $2.13^{\star \star \star}$ & $1.66^{\star \star}$ & $3.52^{\star \star \star}$ & $4.42^{\star \star \star}$ \\
\hline & $(1.46,3.61)$ & $(1.60,2.65)$ & $(1.59,2.84)$ & $(1.19,2.32)$ & $(2.82,4.40)$ & $(3.28,5.96)$ \\
\hline \multirow[t]{2}{*}{ Bangladeshi } & $2.58^{\star \star \star}$ & $2.36^{\star \star \star}$ & $2.39^{\star \star \star}$ & $2.17^{\star}$ & $3.42^{\star \star \star}$ & $4.07^{\star \star \star}$ \\
\hline & $(1.63,4.08)$ & $(1.74,3.21)$ & $(1.81,3.17)$ & $(1.14,4.14)$ & $(2.40,4.87)$ & $(2.36,7.01)$ \\
\hline \multirow[t]{2}{*}{ Chinese } & 1.20 & $0.56^{\star \star \star}$ & $0.44^{\star \star \star}$ & $0.19^{\star \star}$ & 1.15 & 0.66 \\
\hline & $(0.47,3.03)$ & $(0.41,0.76)$ & $(0.30,0.63)$ & $(0.06,0.57)$ & $(0.55,2.39)$ & $(0.29,1.52)$ \\
\hline \multirow[t]{2}{*}{ Age (centred) } & $1.06^{\star \star \star}$ & $1.04^{\star \star \star}$ & $1.04^{\star \star \star}$ & $1.04^{\star \star \star}$ & $1.06^{\star \star \star}$ & $1.05^{\star \star \star}$ \\
\hline & $(1.06,1.07)$ & $(1.03,1.04)$ & $(1.03,1.05)$ & $(1.03,1.05)$ & $(1.05,1.06)$ & $(1.04,1.05)$ \\
\hline
\end{tabular}




\begin{tabular}{|c|c|c|c|c|c|c|}
\hline \multirow[t]{2}{*}{$\mathrm{Age}^{2}$ (centred) } & 1.00 & 1.00 & 1.00 & 1.00 & $1.00^{\star \star \star}$ & $1.00^{\star \star \star}$ \\
\hline & $(1.00,1.00)$ & $(1.00,1.00)$ & $(1.00,1.00)$ & $(1.00,1.00)$ & $(1.00,1.00)$ & $(1.00,1.00)$ \\
\hline \multicolumn{7}{|l|}{ Sex: } \\
\hline \multirow[t]{2}{*}{ Male } & 1.00 & 1.00 & 1.00 & 1.00 & 1.00 & 1.00 \\
\hline & $(1.00,1.00)$ & $(1.00,1.00)$ & $(1.00,1.00)$ & $(1.00,1.00)$ & $(1.00,1.00)$ & $(1.00,1.00)$ \\
\hline \multirow[t]{2}{*}{ Female } & $2.01^{\star \star \star}$ & $1.11^{\star}$ & 1.10 & 1.05 & $1.39^{\star \star \star}$ & $1.42^{\star \star \star}$ \\
\hline & $(1.70,2.38)$ & $(1.01,1.23)$ & $(0.97,1.26)$ & $(0.92,1.21)$ & $(1.30,1.48)$ & $(1.31,1.54)$ \\
\hline Observations & 2,653 & 7,380 & 7,150 & 7,303 & 22,215 & 17,643 \\
\hline Adjusted $R^{2}$ & 0.10 & 0.04 & 0.05 & 0.05 & 0.10 & 0.08 \\
\hline $\mathrm{BIC}$ & $3,377.89(10)$ & $9,178.03(10)$ & $8,779.35$ (11) & $8,371.79$ (11) & $23,796.87(11)$ & $20,559.52(11)$ \\
\hline
\end{tabular}

Note: BIC: Bayesian information criterion.

Significance levels: ${ }^{\star} p<0.05,{ }^{\star \star} p<0.01,{ }^{\star \star \star} p<0.001$. 
4.14 in 2007 to $\mathrm{OR}=4.07,95 \% \mathrm{CI}=2.36-7.01$ in 2015/17). Compared with the odds of LLTI for the White/White British group, Black Caribbean respondents have significantly higher odds in 2009/11 and 2015/17; and Indian respondents have significantly higher odds in 1999, 2009/11 and 2015/17. Chinese respondents have significantly lower odds of LLTI in 1999, 2004 and 2007. Black African respondents have significantly lower odds of LLTI in 2007, but significantly higher odds in 2009/11 and 2015/17. Irish respondents do not have significantly different odds of reporting LLTI compared to the White/White British group in any survey year.

Table 3 presents the cross-sectional logistic regression models for fair or poor self-rated health by ethnicity, age, age-squared, and sex. There are similarly stark ethnic health inequalities which persist over time. Notably, there are significantly higher odds of fair or poor health for Black Caribbean, Indian, Pakistani, and Bangladeshi respondents across all models in most years (with exceptions for Indian and Bangladeshi respondents in 2015/17). The odds of reporting fair or poor self-rated health compared with the White/White British group are at least double for the Pakistani group (95\% CI $=1.51-2.85)$, at least 1.64 times higher $(95 \% \mathrm{CI}=0.94-2.87)$ for the Bangladeshi group, and at least 1.5 times higher $(95 \% \mathrm{CI}=1.09-2.14)$ for the Black Caribbean group in all years. Compared with the odds of fair or poor self-rated health for the White/White British group, Irish respondents have significantly higher odds in 1999 only; Chinese respondents have significantly higher odds in 1999 and 2004 only; and Black African respondents have significantly higher odds in 2004, but non-significant yet lower odds in all other years.

The magnitude of inequalities of LLTI and fair or poor self-rated health tend to fluctuate in adjacent survey years. Over time, the direction of effects suggest that inequalities in LLTI might be marginally increasing, whereas inequalities in fair or poor self-rated health might be marginally decreasing. Due to the differences in data and measurement collections and sample selection strategies, however, we emphasise that these are tentative conclusions about the strength of effects over time. ${ }^{5}$ Nonetheless, these results illustrate a clear persistence of health disadvantage over more than two decades. This health inequality is starkest for Pakistani and Bangladeshi respondents.

In the next stage of the analysis, we additionally adjust for socio-economic position and experiences of racial discrimination (where measures are available). Income, education, and social class are significantly associated with both LLTI and fair or poor self-rated health, whereby people in more advantaged socioeconomic positions tend to have better health outcomes. Although there is a trend for a negative association between experienced racial discrimination and health, the magnitude and statistical significance of the associations differ across datasets. Adjusting for contemporaneous measures of socio-economic position and experienced racial discrimination partially explains ethnic health inequalities, although in most years we continue to observe significantly higher odds for Pakistani and Bangladeshi respondents for both LLTI and self-rated health, and significantly higher odds of fair or poor self-rated health for Black Caribbean and Indian respondents. Tables S3 and S4 in the online supplementary material present the full regression output for all three models in each survey year. 
Table 3. Logistic regression models of fair or poor self-rated health across survey years

\begin{tabular}{|c|c|c|c|c|c|c|}
\hline & $\begin{array}{l}\text { Fourth National } \\
\text { Survey of Ethnic } \\
\text { Minorities 1993/94 }\end{array}$ & $\begin{array}{l}\text { Health } \\
\text { Survey for } \\
\text { England } 1999\end{array}$ & $\begin{array}{c}\text { Health } \\
\text { Survey for } \\
\text { England } 2004\end{array}$ & $\begin{array}{l}\text { Citizenship } \\
\text { Survey } 2007\end{array}$ & $\begin{array}{c}\text { Understanding } \\
\text { Society Wave } 1 \\
2009 / 11\end{array}$ & $\begin{array}{c}\text { Understanding } \\
\text { Society Wave } 7 \\
2015 / 17\end{array}$ \\
\hline & \multicolumn{6}{|c|}{ Odds ratios (95\% confidence intervals) } \\
\hline \multicolumn{7}{|l|}{ Ethnicity: } \\
\hline \multirow[t]{2}{*}{ White/White British } & 1.00 & 1.00 & 1.00 & 1.00 & 1.00 & 1.00 \\
\hline & $(1.00,1.00)$ & $(1.00,1.00)$ & $(1.00,1.00)$ & $(1.00,1.00)$ & $(1.00,1.00)$ & $(1.00,1.00)$ \\
\hline \multirow[t]{2}{*}{ Irish } & 0.86 & $1.23^{\star}$ & 1.01 & 1.00 & 1.01 & 1.38 \\
\hline & $(0.55,1.34)$ & $(1.01,1.49)$ & $(0.80,1.27)$ & $(0.64,1.57)$ & $(0.73,1.40)$ & $(0.87,2.20)$ \\
\hline \multirow[t]{2}{*}{ Black Caribbean } & $2.44^{\star \star \star}$ & $2.51^{\star \star \star}$ & $2.01^{\star \star \star}$ & $1.78^{\star \star \star}$ & $1.63^{\star \star \star}$ & $1.53^{*}$ \\
\hline & $(1.99,2.98)$ & $(2.03,3.11)$ & $(1.57,2.58)$ & $(1.43,2.22)$ & $(1.36,1.96)$ & $(1.09,2.14)$ \\
\hline \multirow[t]{2}{*}{ Black African } & & & $1.47^{\star}$ & 0.84 & 0.99 & 0.97 \\
\hline & & & $(1.06,2.03)$ & $(0.63,1.13)$ & $(0.79,1.26)$ & $(0.64,1.47)$ \\
\hline \multirow[t]{2}{*}{ Indian } & $1.98^{\star \star \star}$ & $2.57^{\star \star \star}$ & $2.14^{\star \star \star}$ & $1.70^{\star \star \star}$ & $1.39^{\star \star \star}$ & 1.08 \\
\hline & $(1.47,2.67)$ & $(2.02,3.27)$ & $(1.71,2.68)$ & $(1.37,2.12)$ & $(1.15,1.68)$ & $(0.83,1.39)$ \\
\hline \multirow[t]{2}{*}{ Pakistani } & $3.90^{\star \star \star}$ & $4.70^{\star \star \star}$ & $3.56^{\star \star \star}$ & $2.76^{\star \star \star}$ & $2.71^{\star \star \star}$ & $2.08^{\star \star \star}$ \\
\hline & $(3.08,4.92)$ & $(3.60,6.15)$ & $(2.67,4.73)$ & $(2.02,3.77)$ & $(2.17,3.37)$ & $(1.51,2.85)$ \\
\hline \multirow[t]{2}{*}{ Bangladeshi } & $5.22^{\star \star \star}$ & $7.09^{\star \star \star}$ & $5.80^{\star \star \star}$ & $2.30^{\star \star}$ & $2.71^{\star \star \star}$ & 1.64 \\
\hline & $(2.81,9.70)$ & $(5.33,9.43)$ & $(4.17,8.06)$ & $(1.22,4.33)$ & $(1.94,3.78)$ & $(0.94,2.87)$ \\
\hline \multirow[t]{2}{*}{ Chinese } & 1.57 & $1.56^{\star}$ & $1.43^{\star}$ & 1.28 & 0.58 & 0.87 \\
\hline & $(0.80,3.08)$ & $(1.09,2.23)$ & $(1.02,1.99)$ & $(0.58,2.81)$ & $(0.27,1.23)$ & $(0.39,1.95)$ \\
\hline
\end{tabular}


Table 3. (Continued)

\begin{tabular}{|c|c|c|c|c|c|c|}
\hline & $\begin{array}{l}\text { Fourth National } \\
\text { Survey of Ethnic } \\
\text { Minorities 1993/94 }\end{array}$ & $\begin{array}{c}\text { Health } \\
\text { Survey for } \\
\text { England } 1999\end{array}$ & $\begin{array}{c}\text { Health } \\
\text { Survey for } \\
\text { England } 2004\end{array}$ & $\begin{array}{l}\text { Citizenship } \\
\text { Survey } 2007\end{array}$ & $\begin{array}{c}\text { Understanding } \\
\text { Society Wave } 1 \\
2009 / 11\end{array}$ & $\begin{array}{c}\text { Understanding } \\
\text { Society Wave } 7 \\
2015 / 17\end{array}$ \\
\hline \multirow[t]{2}{*}{ Age (centred) } & $1.03^{\star \star *}$ & $1.04^{\star \star \star}$ & $1.04^{\star \star *}$ & $1.04^{\star \star \star}$ & $1.03^{\star \star *}$ & $1.03^{\star * *}$ \\
\hline & $(1.02,1.04)$ & $(1.04,1.05)$ & $(1.03,1.05)$ & $(1.03,1.05)$ & $(1.03,1.04)$ & $(1.02,1.03)$ \\
\hline \multirow[t]{2}{*}{ Age $^{2}$ (centred) } & 1.00 & $1.00^{\star}$ & 1.00 & 1.00 & 1.00 & 1.00 \\
\hline & $(1.00,1.00)$ & $(1.00,1.00)$ & $(1.00,1.00)$ & $(1.00,1.00)$ & $(1.00,1.00)$ & $(1.00,1.00)$ \\
\hline \multirow[t]{2}{*}{ Male } & 1.00 & 1.00 & 1.00 & 1.00 & 1.00 & 1.00 \\
\hline & $(1.00,1.00)$ & $(1.00,1.00)$ & $(1.00,1.00)$ & $(1.00,1.00)$ & $(1.00,1.00)$ & $(1.00,1.00)$ \\
\hline \multirow[t]{2}{*}{ Female } & $1.34^{*}$ & 1.06 & 1.10 & 0.99 & 1.01 & $1.11^{\star}$ \\
\hline & $(1.09,1.65)$ & $(0.96,1.17)$ & $(0.97,1.25)$ & $(0.87,1.13)$ & $(0.95,1.08)$ & $(1.02,1.21)$ \\
\hline
\end{tabular}

Note: BIC: Bayesian information criterion.

Significance levels: ${ }^{\star} p<0.05,{ }^{\star \star} p<0.01,{ }^{\star \star \star} p<0.001$. 
We compare the marginal effects of the health outcomes to assess the effects of socio-economic position and experienced racial discrimination on ethnic inequalities for LLTI (Figure 1) and fair or poor self-rated health (Figure 2). Attenuations refer to the changes in relative probabilities (and narrowing of inequalities) between each minoritised group and the White/White British comparison group after adjusting for age, age-squared, and sex (Model 1), additionally adjusting for socio-economic position (Model 2), and additionally adjusting for experienced racial discrimination (Model 3). ${ }^{6}$ The changes in relative probabilities are expressed in percentage points.

Adjusting for socio-economic position has a significant and substantial attenuating effect on inequalities in all survey years for Pakistani respondents (7-10 percentage points for LLTI and 8-14 percentage points for fair or poor self-rated health) and Bangladeshi respondents (7-14 percentage points for LLTI and 7-19 percentage points for fair or poor self-rated health). There are more modest attenuating effects on inequalities in health for Black Caribbean respondents (1-4 percentage points for LLTI and 1-7 percentage points for fair or poor self-rated health) and Indian respondents (0-5 percentage points for LLTI and 1-6 percentage points for fair or poor self-rated health). The attenuations are significant in all years except 2009/11 for Indian respondents, and 2015/17 for both Black Caribbean and Indian respondents. The changes in inequalities in health are significant in all survey years for Black African respondents, however, the direction of effects are more nuanced. Where relative probabilities are higher than the White/White British group, inequalities in health are attenuated, but where relative probabilities are lower, inequalities in health increase between Models 1 and 2. Attenuations are significant for Chinese respondents in 1999 for LLTI and 2009/11 for both outcomes, and attenuations are negligible and non-significant for Irish respondents across all survey years.

Additionally adjusting for experienced racial discrimination has minimal substantive impact on the predicted probabilities for all ethnic groups (between 0 and 3 percentage points in all years). However, these slight attenuations are significant in models of LLTI for Indian respondents in 1993/94, and in models of self-rated health for Black Caribbean, Black African, Indian, Pakistani, and Bangladeshi respondents in 2007.

To assess the changes and magnitude of ethnic health inequalities in later life, we estimate interaction effects for ethnicity, age, and age-squared in each of the datasets. The interaction effects are significant for both health outcomes, with $p<0.001$ in all surveys except the Fourth National Survey of Ethnic Minorities. ${ }^{7}$ The interaction effects in each survey individually demonstrate patterns of increasing ethnic health inequalities in the oldest ages, with the greatest inequalities for Pakistani and Bangladeshi respondents compared with White/White British respondents. When interaction effects are calculated separately in each survey year, the trajectories of health inequalities in older ages are broadly similar for Black Caribbean, Indian and Pakistani respondents, but variations are observable for Black African, Bangladeshi and Chinese respondents (see Figures S1 and S2 in the online supplementary material). These groups are also the most underrepresented in the survey data, particularly in the oldest ages. 


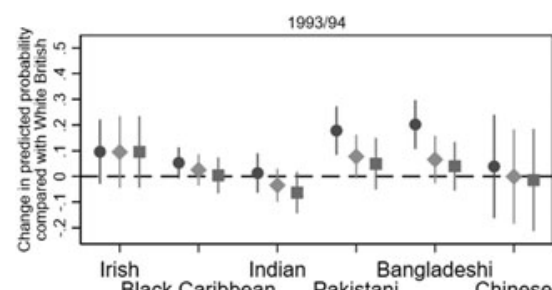

Irish Indian
Black Caribbean Pakistani ${ }_{\text {Chinese }}^{\text {Bangleshi }}$

2007

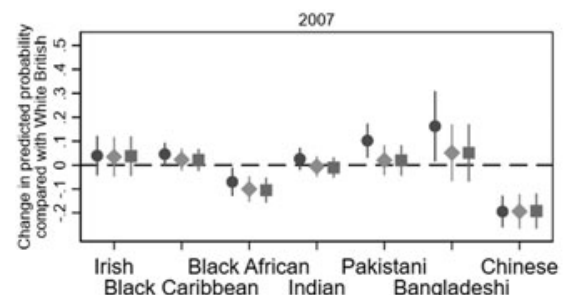

Irish Black African Pakistani Chine
Black Caribbean Indian Bangladeshi

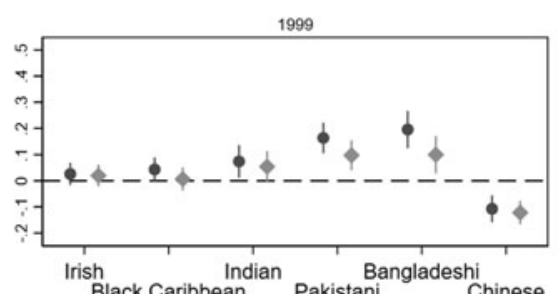

Indian
Black Caribbean Pakistani $\quad$ Chinese

2009/11

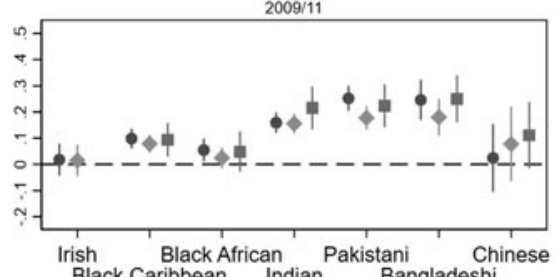

Brish Caribbean Indian Bangladeshi

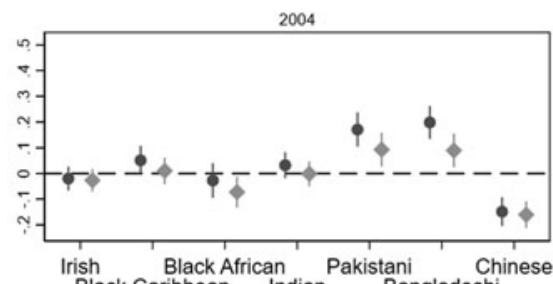

Irish Black African Pakistani Chi
Black Caribbean Indian

2015/17

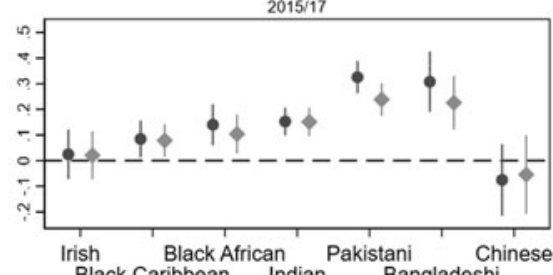

Black Caribbean Indian Bangladeshi

\section{Model $1>$ Model $2 \square$ Model 3}

Figure 1. Relative probabilities of limiting long-term illness by ethnicity with $95 \%$ confidence intervals.

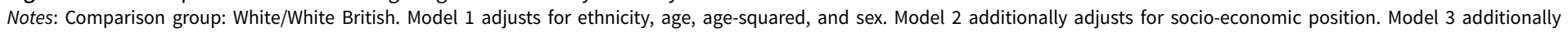
adjusts for racism and racial discrimination. Note that Model 3 for Understanding Society Wave 1 is estimated on the extra five-minute sample only ( $\mathrm{n}=2,730$ ).

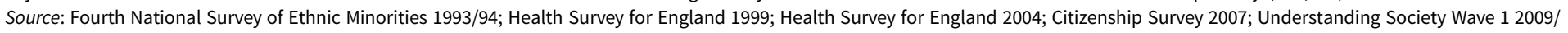
11; and Understanding Society Wave 7 2015/17. 

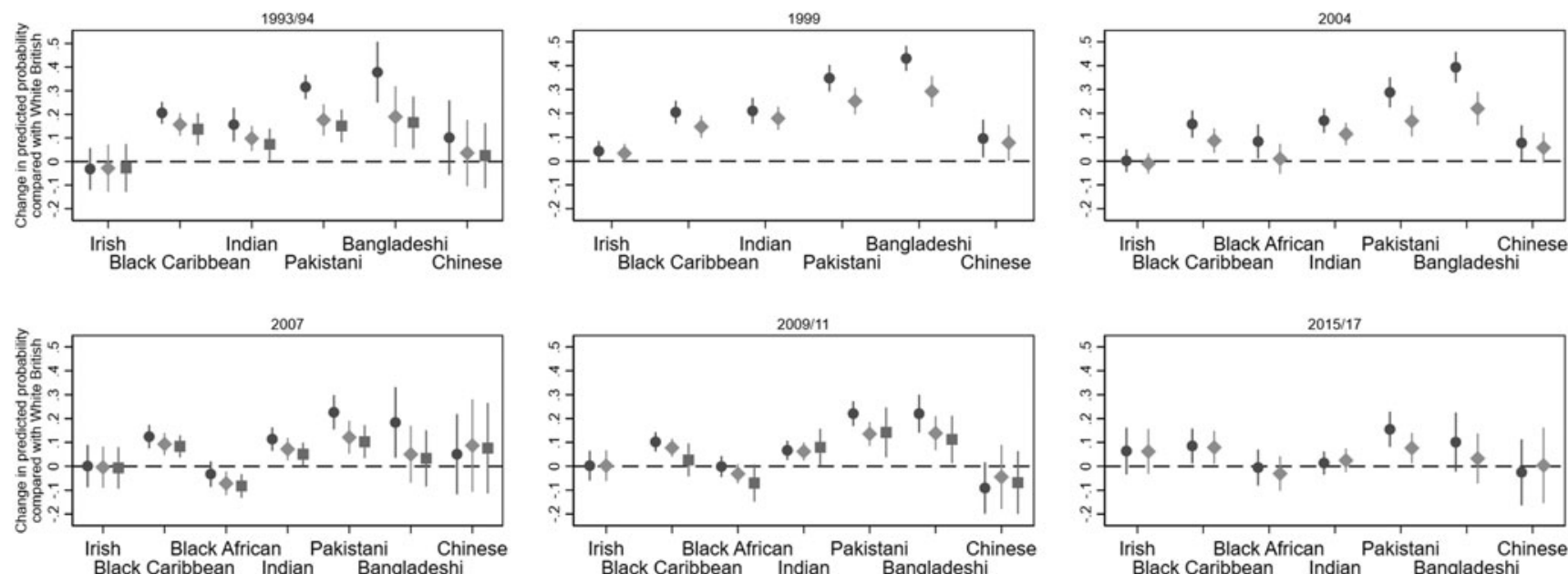

- Model $1 \diamond$ Model 2 Model 3

Figure 2. Relative probabilities of fair or poor self-rated health by ethnicity with $95 \%$ confidence intervals.

Notes: Comparison group: White/White British. Model 1 adjusts for ethnicity, age, age-squared and sex. Model 2 additionally adjusts for socio-economic position. Model 3 additionally adjusts for racism and racial discrimination. Note that Model 3 for Understanding Society Wave 1 is estimated on the extra five-minute sample only ( $\mathrm{n}=2,730)$.

Source: Fourth National Survey of Ethnic Minorities 1993/94; Health Survey for England 1999; Health Survey for England 2004; Citizenship Survey 2007; Understanding Society Wave 1 2009/

11; and Understanding Society Wave 7 2015/17. 


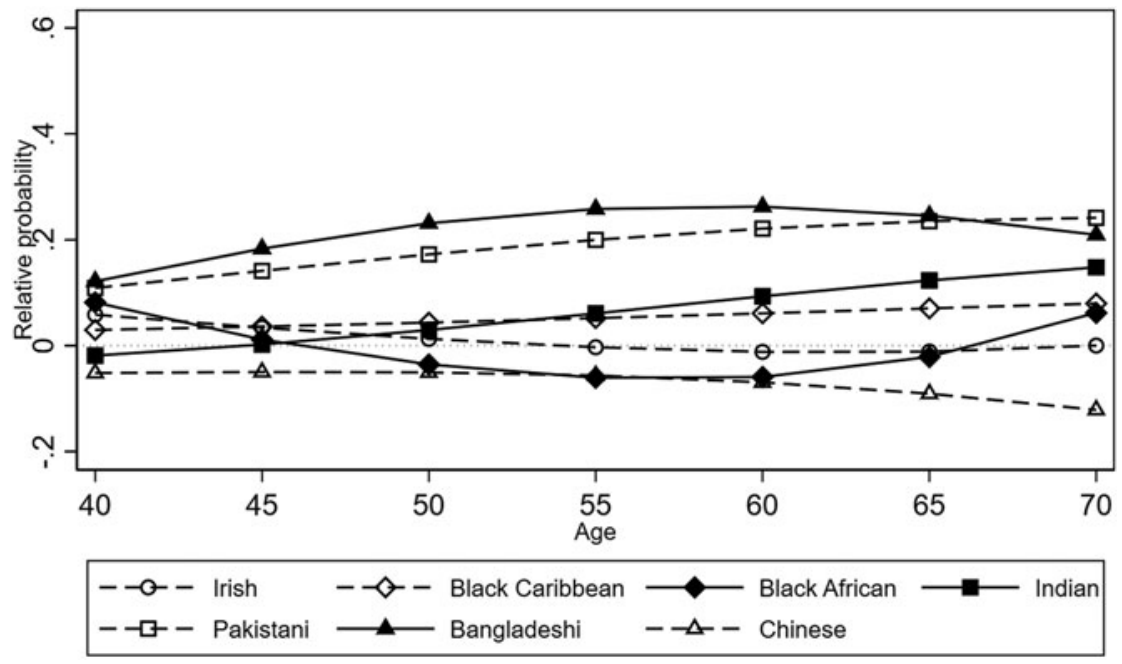

Figure 3. Relative probabilities of limiting long-term illness: interaction of ethnicity, age, and agesquared (indicative trends).

Notes: Comparison group: White/White British. Model is indicative of broader trends of ethnic inequalities in limiting long-term illness in later life. Model includes main effects of ethnicity, age, age-squared, sex, and survey year, and interaction effects of ethnicity, age, and age-squared.

Source: Data are pooled from the Fourth National Survey of Ethnic Minorities 1993/4; Health Survey for England 1999; Health Survey for England 2004; Citizenship Survey 2007; Understanding Society Wave 1, 2009/11; and Understanding Society Wave 7 2015/17. Where respondents are present in both Waves 1 and 7 of Understanding Society, data are taken from Wave 1 only.

We re-estimated the interaction effects on a pooled dataset to work with increased sample sizes and associated statistical power. This is an exploratory approach, and the results are indicative of broader trends of ethnic health inequalities which we might observe given more adequate sample sizes of older ethnic minority people. The model includes the main effects of ethnicity, age, age-squared, sex, and survey year, and the interaction effects of ethnicity, age, and age-squared. The model is weighted using corresponding survey weights, with scaled adjustments to ensure that ethnicity is equally represented across surveys (i.e. that one survey does not have overriding influence). Where Understanding Society respondents were present in both Waves 1 and 7, we only used the data from Wave 1 for this model.

Figure 3 presents the indicative trends for LLTI and Figure 4 presents the indicative trends for fair or poor self-rated health. Inequalities tend to be greater for fair or poor self-rated health than for LLTI. Inequalities in health are greatest in the oldest ages for Pakistani, Bangladeshi, Black Caribbean, and Indian respondents, compared with the White/White British group. The differences in probabilities compared with the White/White British group tend to be consistently highest for Pakistani and Bangladeshi respondents, and more modest, but with steeper slopes, for Black Caribbean and Indian respondents. The trajectory of ethnic health inequalities is more nuanced for Black African and Chinese respondents. For fair or poor self-rated health, Black African and Chinese respondents have very similar 


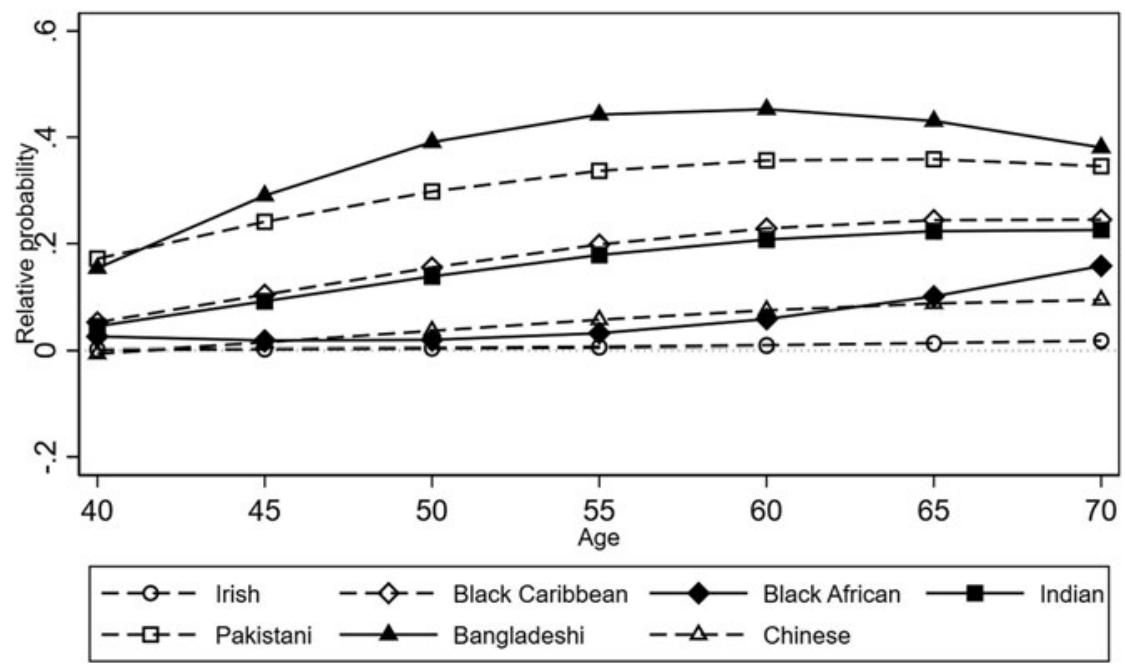

Figure 4. Relative probabilities of fair or poor self-rated health: interaction of ethnicity, age, and agesquared (indicative trends).

Notes: Comparison group: White/White British. Model is indicative of broader trends of ethnic inequalities in fair or poor self-rated health in later life. Model includes main effects of ethnicity, age, age-squared, sex, and survey year, and interaction effects of ethnicity, age, and age-squared.

Source: Data are pooled from the Fourth National Survey of Ethnic Minorities 1993/4; Health Survey for England 1999; Health Survey for England 2004; Citizenship Survey 2007; Understanding Society Wave 1, 2009/11 and Understanding Society Wave 7 2015/17. Where respondents are present in both Waves 1 and 7 of Understanding Society, data are taken from Wave 1 only.

or marginally higher probabilities of fair or poor self-rated health compared with the White/White British respondents, which are greatest for the oldest respondents. For LLTI, Chinese respondents have consistently lower probabilities of LLTI than the White/White British respondents, and Black African respondents have lower probabilities of LLTI in mid-life, but higher probabilities in the youngest and oldest ages. Irish respondents have very similar probabilities of LLTI and fair or poor selfrated health as White/White British respondents across all ages.

\section{Discussion and conclusions}

In this paper, we have taken a novel approach to examining inequalities over time by harmonising a wide range of social survey data spanning a period of more than 20 years. We find a clear persistence of ethnic inequalities in health in later life. There are stark and significant ethnic inequalities in LLTI and self-rated health for people aged 40 and over in the 1990s, 2000s, and the 2010s. Ethnic health inequalities tend to be largest in older ages, and are partially explained by contemporaneous measures of socio-economic position and experienced racial discrimination. The findings correspond with previous research examining ethnic inequalities in LLTI and self-rated health in adulthood (Harding and Balarajan, 2000; Nazroo, 2001b; Bécares, 2015; Darlington et al., 2015). Our findings build upon previous studies by specifically focusing on later life and by providing 
much needed evidence on the nature and persistence of ethnic inequalities in health.

Our results demonstrate that most minoritised ethnic groups have much poorer health profiles compared with the White/White British group. We find that older Black Caribbean, Indian, and particularly Pakistani and Bangladeshi respondents are worst affected by ill-health. Ethnic health inequalities persist over time and are clearly observable in each survey year. These findings chime with previous work using Census data in 1991, 2001, and 2011 (Bécares, 2015; Stopforth et al., forthcoming). Ethnic inequalities in health also seem to be greater for older respondents, as evidenced by significant interaction effects between ethnicity, age, and age-squared. Approaches aimed at reducing ethnic inequalities need to address adequately the more nuanced magnitude and trajectory of ethnic inequalities in health in later life.

There are associations between less advantaged socio-economic positions and poorer health, and between experienced racial discrimination and poorer health. This complements previous research examining the importance of these structural determinants of health (Williams, 1999; Nazroo, 2003; Bécares et al., 2009; Hudson et al., 2013; Darlington et al., 2015). Ethnic inequalities in LLTI and fair or poor self-rated health are partially explained by contemporaneous measures of socioeconomic disadvantage and experienced racial discrimination. Current socioeconomic position modestly attenuates the effect sizes for Black Caribbean and Indian respondents, and more substantially attenuates the effect sizes for Pakistani and Bangladeshi respondents. We also observed that Pakistani and Bangladeshi groups were severely under-represented in the most advantaged socioeconomic positions. It is therefore plausible that the stark and consistent socioeconomic disadvantage experienced by Pakistani and Bangladeshi respondents in all survey years may therefore explain inequalities in health outcomes to a greater degree.

Experiences or anticipation of racism and discrimination tend to have more minor substantive attenuations on ethnic inequalities in both health outcomes in our statistical models. The measures of experienced racial discrimination available in the surveys analysed refer to a specific period in the recent past, which is likely to underestimate the fuller extent of racism on health outcomes compared with measures which can identify accumulation of racism and discrimination over time, domains and generations (Wallace et al., 2016). Racism is not only experienced interpersonally, but also operates through socio-economic inequality. It is plausible that much of the effect of racism in our models is acting indirectly through socioeconomic inequalities, representing a more complex mechanism of structural inequalities.

We also note that the variables available for socio-economic position and racism are imperfect. The measures can only partially cover the full extent of socioeconomic position and racism in the survey context. The measures are crosssectional, referring to one point in time, and cannot adequately capture lifecourse accumulation of disadvantage on poorer health outcomes. We do, however, observe clear ethnic inequalities in later life. Prolonged exposure to disadvantage and discrimination will have longer-term effects than contemporaneous, cross-sectional measures can illustrate. Our results are associational rather than causal, and can 
only indirectly test the effects of cumulative disadvantage and experienced racial discrimination. We theorise that the core explanations of the stark ethnic health inequalities we observe in this paper are a product of accumulation of disadvantage and exposure to racism and discrimination over the lifecourse. The evidence generated by our repeated cross-sectional models supports this theoretical approach. However, there is currently no suitable UK longitudinal survey data to directly test this hypothesis.

A key limitation in the present study is the restrictive sample sizes and associated statistical power in any one cross-sectional survey to examine ethnic inequalities for the oldest cohorts. Given this, we would conjecture that our cross-sectional results will underestimate the full extent of inequalities in later life. The lack of statistical power is particularly pertinent when calculating interaction effects. We took an exploratory approach to a data problem by calculating interaction effects on pooled data. The benefit of the pooled model was to work with much larger sample sizes than in any one dataset. However, we note the methodological limitations of pooling data, and stress that these analyses are exploratory, and the patterns are indicative of broader trends of inequalities in older age that we might observe if we were to have access to adequate sample sizes of older ethnic minority people in existing data.

In this paper, we have highlighted the data problem encountered when investigating ethnic inequalities in health for older people in England. There are large data and evidence gaps present to examine, monitor, and explain ethnic inequalities in health and socio-economic circumstances for older people in the UK, and the interrelated and cumulative effects of socio-economic position and racism over the lifecourse (Bécares et al., 2020). This marginalisation in UK research is prevalent in both the gerontological field and ethnicity studies (Torres, 2015). Victor et al. (2021) outlined the need for gerontological research to more suitably reflect the increasing ethnic diversity of ageing populations. The lack of suitable data to study ethnic inequalities in the ageing process plays a large part in this marginalisation, as studies do not tend to have appropriate sample sizes of older respondents from minoritised ethnic groups to conduct robust analyses (Bécares et al., 2020). In the UK, we are privileged with access to large-scale, high-quality health and social survey data which enable suitable investigations of ageing for older White British people. These include birth cohort and longitudinal studies, such as the 1946 National Survey of Health and Development, the 1958 National Child Development Study, the 1970 British Cohort Study, and the English Longitudinal Study of Ageing. However, these data tend not to allow either robust analyses of older ethnic minority people specifically, or to examine period and cohort differences.

By presenting analyses from a number of different data sources we can partially overcome some of the data limitations. We remain mindful of the limitations of using cross-sectional social surveys to study ethnic inequalities for older people, particularly in accurate measurement of lifecourse accumulation of social and economic disadvantage, and understanding changes as people age. Nonetheless, we find clear ethnic inequalities in health which persist across the multiple data sources and health outcomes. Our results make an important contribution to the growing evidence base on ethnic health inequalities in later life over time in the UK. 
The approach we present here needs to be reinforced with robust data collection in order to understand fully ethnic inequalities in health in later life (Bécares et al., 2020). Future data collection endeavours must focus not only on current socioeconomic position and experiences of racism, but encompass a longer-term approach to understand better the accumulation of disadvantage and its effects on poorer health outcomes for older ethnic minority people compared with the White British group. Improving longitudinal data resources for older ethnic minority people is a critical area of future investment. This much-needed data would enable researchers to test directly, and better understand, the effects of the accumulation of lifecourse disadvantage on ethnic inequalities in health outcomes in later life.

Supplementary material. The supplementary material for this article can be found at https://doi.org/10. 1017/S0144686X2100146X

Acknowledgements. We gratefully acknowledge the participants of the Fourth National Survey of Ethnic Minorities, the Health Surveys for England, the Citizenship Survey, and Understanding Society. We would also like to thank the two anonymous reviewers for their helpful comments on an earlier draft of this paper.

Financial support. This work was supported by the Nuffield Foundation (LB, award WEL/43881), but the views expressed are those of the authors and not necessarily those of the Foundation.

Conflict of interest. The authors declare no conflicts of interest.

\section{Notes}

1 We only use Waves 1 and 7 of Understanding Society to incorporate new respondents from the refresher Immigrant and Ethnic Minority Boost (IEMB) sample. The IEMB sample was introduced in Wave 6, but IEMB respondents were not asked about limiting long-term illness (LLTI) in this wave.

2 In the Fourth National Survey of Ethnic Minorities, all respondents were asked about having a long-standing illness, and if this limits the paid work they can do or would like to do. A sub-sample of respondents were asked about LLTI which affects a list of typical daily activities. We use the latter measure, as it is more compatible with the definitions of LLTI present in the other surveys, although our sample size is reduced as a result. 3 Where an NS-SEC position cannot be identified, respondents are coded as 'no class' and retained in the analyses. The reasons for not having an NS-SEC position are numerous, e.g. not being in employment, being long-term unemployed, or not providing enough information to derive a social class position. Due to the large within-group heterogeneity, we do not place emphasis on the interpretation of this category.

4 To facilitate useful comparisons across surveys and ethnic groups within space restrictions, in Table 1 we only present the percentages in the most advantaged socio-economic positions (i.e. highest income quintile, degree-level education, and managerial or professional occupations), and percentages for those who have experienced racial attacks, fear racial harassment, or have avoided places due to harassment. These measures are, however, categorical in our models.

5 We formally test the differences in coefficients across surveys using the method outlined in Mize et al. (2019) and find that adjacent survey years do not have significantly different results.

6 For the 2009/11 results, only respondents in the extra five-minute sample are asked about racism and discrimination, resulting in a heavily reduced sample size for Model $3(n=2,730)$ compared with Models 1 and $2(\mathrm{n}=22,215)$. We re-estimated Models 2 and 3 on the extra five-minute sample to assess changes in probability for $2009 / 11$ as mentioned in the text.

7 We test for global significance using Wald tests.

\section{References}

Bartley M, Sacker A and Clarke P (2004) Employment status, employment conditions, and limiting illness: prospective evidence from the British Household Panel Survey 1991-2001. Journal of Epidemiology and Community Health 58, 501-506. 
Bécares L (2015) Which ethnic groups have the poorest health? In Jivraj S and Simpson L (eds). Ethnic Identity and Inequalities in Britain: The Dynamics of Diversity. Bristol, UK: Policy Press, pp. 123-139.

Bécares L, Stafford M and Nazroo J (2009) Fear of racism, employment and expected organizational racism: their association with health. European Journal of Public Health 19, 504-510.

Bécares L, Nazroo J and Kelly Y (2015) A longitudinal examination of maternal, family, and area-level experiences of racism on children's socioemotional development: patterns and possible explanations. Social Science \& Medicine 142, 128-135.

Bécares L, Kapadia D and Nazroo J (2020) Neglect of older ethnic minority people in UK research and policy. BMJ 368, m212.

Berthoud RG, Prior G, Smith P and Modood T (1997) Fourth National Survey of Ethnic Minorities 1993-1994 (Data collection). Colchester, UK: UK Data Service. SN:3685.

Bhopal R, Hayes L, White M, Unwin N, Harland J, Ayis S and Alberti G (2002) Ethnic and socioeconomic inequalities in coronary heart disease, diabetes and risk factors in Europeans and South Asians. Journal of Public Health Medicine 24, 95-105.

Brynin M and Longhi S (2015) The Effect of Occupation on Poverty Among Ethnic Minority Groups. London: Joseph Rowntree Foundation.

Clark K and Shankley W (2020) Ethnic minorities in the labour market in Britain. In Byrne B, Alexander C, Khan O, Nazroo J and Shankley W (eds). Ethnicity, Race and Inequality in the UK: State of the Nation. Bristol, UK: Policy Press, pp. 127-147.

Connelly R, Gayle V and Lambert PS (2016) Statistical modelling of key variables in social survey data analysis. Methodological Innovations 9, 1-17.

Dannefer D (2003) Cumulative advantage/disadvantage and the life course: cross-fertilizing age and social science theory. Journals of Gerontology: Psychological Sciences and Social Sciences 58B, S327-S337.

Darlington F, Norman P, Ballas D and Exeter DJ (2015) Exploring ethnic inequalities in health: evidence from the Health Survey for England, 1998-2011. Diversity and Equality in Health and Care 12, 54-65.

Dearden L and Sibieta L (2010) Ethnic inequalities in child outcomes. In Hansen K, Joshi H and Dex S (eds). Children of the 21st Century, Vol. 2. Bristol, UK: Bristol University Press, pp. 169-184.

Department for Communities and Local Government, Race, Cohesion and Faith Research Unit, National Centre for Social Research (2019) Citizenship Survey, 2007-2008, 7th Edn (Data collection). Colchester, UK: UK Data Service. SN:5739.

Evandrou M (2000) Ethnic inequalities in health in later life. Health Statistics Quarterly 8, 20-28.

Evandrou M, Falkingham J, Feng Z $\mathbf{Z}$ and Vlachantoni A (2016) Ethnic inequalities in limiting health and self-reported health in later life revisited. Journal of Epidemiology and Community Health 70, 653-662.

Finney N and Harries B (2013) Understanding ethnic inequalities in housing: analysis of the 2011 Census. Race Equality Foundation, London, Better Housing Briefing 23.

Forde AT, Crookes DM, Suglia SF and Demmer RT (2019) The weathering hypothesis as an explanation for racial disparities in health: a systematic review. Annals of Epidemiology 33, 1-18.e3.

Gee GC, Walsemann KM and Brondolo E (2012) A life course perspective on how racism may be related to health inequities. American Journal of Public Health 102, 967-974.

Gee GC, Hing A, Mohammed S, Tabor DC and Williams DR (2019) Racism and the life course: taking time seriously. American Journal of Public Health 109, S43-S47.

Geronimus AT (1992) The weathering hypothesis and the health of African-American women and infants: evidence and speculations. Ethnicity \& Disease 2, 207-221.

Hackett RA, Ronaldson A, Bhui K, Steptoe A and Jackson SE (2020) Racial discrimination and health: a prospective study of ethnic minorities in the United Kingdom. BMC Public Health 20, 1652.

Harding S and Balarajan R (2000) Limiting long-term illness among Black Caribbeans, Black Africans, Indians, Pakistanis, Bangladeshis and Chinese born in the UK. Ethnicity \& Health 5, 41-46.

Hudson DL, Puterman E, Bibbins-Domingo K, Matthews KA and Adler NE (2013) Race, life course socioeconomic position, racial discrimination, depressive symptoms and self-rated health. Social Science \& Medicine 97, 7-14.

Jivraj S and Khan $\mathbf{O}$ (2015) How likely are people from minority ethnic groups to live in deprived neighbourhoods? In Jivraj S and Simpson L (eds). Ethnic Identity and Inequalities in Britain: The Dynamics of Diversity. Bristol, UK: Policy Press, pp. 199-213. 
Kapadia D, Nazroo J and Clark K (2015) Have ethnic inequalities in the labour market persisted? In Jivraj S and Simpson L (eds). Ethnic Identity and Inequalities in Britain: The Dynamics of Diversity. Bristol, UK: Policy Press, pp. 161-179.

Karlsen S and Nazroo JY (2002) Relation between racial discrimination, social class, and health among ethnic minority groups. American Journal of Public Health 92, 624-631.

Karlsen S and Nazroo JY (2004) Fear of racism and health. Journal of Epidemiology and Community Health 58, 1017-1018.

Karlson KB, Holm A and Breen R (2012) Comparing regression coefficients between same-sample nested models using logit and probit: a new method. Sociological Methodology 42, 286-313.

Kelly Y, Panico L, Bartley M, Marmot M, Nazroo J and Sacker A (2009) Why does birthweight vary among ethnic groups in the UK? Findings from the Millennium Cohort Study. Journal of Public Health 31, 131-137.

Kendig H and Nazroo J (2016) Life course influences on inequalities in later life: comparative perspectives. Journal of Population Ageing 9, 1-7.

Li Y and Heath A (2020) Persisting disadvantages: a study of labour market dynamics of ethnic unemployment and earnings in the UK (2009-2015). Journal of Ethnic and Migration Studies 46, 857-878.

Lievesley N (2010) The Future Ageing of the Ethnic Minority Population of England and Wales: Older BME People and Financial Inclusion Report. London: Runnymede Trust and the Centre for Policy on Ageing.

Maheswaran H, Kupek E and Petrou S (2015) Self-reported health and socio-economic inequalities in England, 1996-2009: repeated national cross-sectional study. Social Science \& Medicine 136/137, 135-146.

Marmot M (2010) Fair Society, Healthy Lives: The Marmot Review. London: Marmot Review.

Marmot M (2020) Health equity in England: the Marmot review 10 years on. BMJ 368, m693.

Martinson ML, McLanahan S and Brooks-Gunn J (2012) Race/ethnic and nativity disparities in child overweight in the United States and England. Annals of the American Academy of Political and Social Science 643, 219-238.

Mize TD, Doan L and Long JS (2019) A general framework for comparing predictions and marginal effects across models. Sociological Methodology 49, 152-189.

National Centre for Social Research and UCL Department of Epidemiology and Public Health (2010a) Health Survey for England 1999, 4th Edn (Data collection). Colchester, UK: UK Data Service. SN:4365.

National Centre for Social Research and UCL Department of Epidemiology and Public Health (2010b) Health Survey for England 2004, 2nd Edn (Data collection). Colchester, UK: UK Data Service. SN:5439.

Nazroo J (1997) Health and health services. In Modood T, Berthoud R and Lakey J (eds). Ethnic Minorities in Britain: Diversity and Disadvantage: The Fourth National Survey of Ethnic Minorities. London: Policy Studies Institute, pp. 224-251.

Nazroo JY (2001a) Ethnicity, Class and Health. London: Policy Studies Institute.

Nazroo JY (2001b) South Asian people and heart disease: an assessment of the importance of socioeconomic position. Ethnicity \& Disease 11, 401-411.

Nazroo JY (2003) The structuring of ethnic inequalities in health: economic position, racial discrimination, and racism. American Journal of Public Health 93, 277-284.

Nazroo J (2004) Ethnic disparities in aging health: what can we learn from the United Kingdom? In Anderson NB, Bulatao RA and Cohen B (eds). Critical Perspectives on Racial and Ethnic Differences in Health in Late Life. Washington, DC: The National Academies Press, pp. 677-702.

Office for National Statistics (2015) Family Spending in the UK: Calendar Year 2014. London: Office for National Statistics.

Panico L, Bartley M, Marmot M, Nazroo JY, Sacker A and Kelly YJ (2007) Ethnic variation in childhood asthma and wheezing illnesses: findings from the Millennium Cohort Study. International Journal of Epidemiology 36, 1093-1102.

Phillipson C (2015) Placing ethnicity at the centre of studies of later life: theoretical perspectives and empirical challenges. Ageing \& Society 35, 917-934.

Rose D and Pevalin DJ (2003) A Researcher's Guide to the National Statistics Socio-economic Classification. London: Sage.

Scambler G (2012) Health inequalities. Sociology of Health \& Illness 34, 130-146.

Shankley W and Finney N (2020) Ethnic minorities and housing in Britain. In Byrne B, Alexander C, Khan O, Nazroo J and Shankley W (eds). Ethnicity, Race and Inequality in the UK: State of the Nation. Bristol, UK: Policy Press, pp. 149-166. 
StataCorp (2019) Stata Statistical Software: Release 16. College Station, TX: StataCorp.

Stopforth S, Bécares L, Kapadia D and Nazroo J (forthcoming) A life course approach to understanding ethnic health inequalities in later life: an example using the United Kingdom as national context. In Nico M and Pollock G (eds), The Routledge Handbook of Contemporary Inequalities and the Life Course. Abingdon, UK: Routledge.

Torres S (2015) Expanding the gerontological imagination on ethnicity: conceptual and theoretical perspectives. Ageing \& Society 35, 935-960.

University of Essex, Institute for Social and Economic Research (2020) Understanding Society: Waves 1-9, 2009-2018 and Harmonised BHPS: Waves 1-18, 1991-2009, 12th Edn (Data collection). Colchester, UK: UK Data Service. SN:6614.

Victor CR, Burholt V and Martin W (2012) Loneliness and ethnic minority elders in Great Britain: an exploratory study. Journal of Cross-cultural Gerontology 27, 65-78.

Victor CR, Dobbs C, Gilhooly K and Burholt V (2021) Loneliness in mid-life and older adults from ethnic minority communities in England and Wales: measure validation and prevalence estimates. European Journal of Ageing 8, 5-16.

Wallace S, Nazroo J and Bécares L (2016) Cumulative effect of racial discrimination on the mental health of ethnic minorities in the United Kingdom. American Journal of Public Health 106, 1294-1300.

Watkinson RE, Sutton M and Turner AJ (2021) Ethnic inequalities in health-related quality of life among older adults in England: secondary analysis of a national cross-sectional survey. The Lancet Public Health 6, e145-e154.

Williams DR (1999) Race, socioeconomic status, and health. The added effects of racism and discrimination. Annals of the New York Academy of Sciences 896, 173-188.

Williams DR and Mohammed SA (2013) Racism and health I: pathways and scientific evidence. American Behavioral Scientist 57, 1-19.

Wohland P, Rees P, Nazroo J and Jagger C (2015) Inequalities in healthy life expectancy between ethnic groups in England and Wales in 2001. Ethnicity \& Health 20, 341-353.

Zwysen W and Longhi S (2018) Employment and earning differences in the early career of ethnic minority British graduates: the importance of university career, parental background and area characteristics. Journal of Ethnic and Migration Studies 44, 154-172.

Cite this article: Stopforth S, Kapadia D, Nazroo J, Bécares L (2021). Ethnic inequalities in health in later life, 1993-2017: the persistence of health disadvantage over more than two decades. Ageing \& Society 1-29. https://doi.org/10.1017/S0144686X2100146X 